\title{
First-principles study of substitutional carbon pair and Stone-Wales defect complexes in boron nitride nanotubes
}

\author{
Gunn Kim, ${ }^{1}$ Jinwoo Park, ${ }^{1}$ and Suklyun Hong ${ }^{1}$ \\ ${ }^{1}$ Department of Physics and Graphene Research Institute, Sejong University, Seoul 143-747, Korea
}

(Dated: October 25, 2018)

\begin{abstract}
Using density functional theory, we study physical properties of boron nitride nanotubes (BNNTs) with the substitutional carbon pair defect. We also consider the Stone-Wales (SW) rearrangement of the C-C pair defect in the BNNT. The formation energy of an SW defect of the carbon dimer is approximately $3.1 \mathrm{eV}$ lower than that of the SW-transformed B-N pair in the undoped BNNT. The activation energies show that the SW defect in the C-doped BNNT may be experimentally observed with a higher probability than in the undoped BNNT. Finally, we discuss the localized states originating from the carbon pair impurities.
\end{abstract}

As a wide-band-gap nanomaterial[1] [3], hexagonal boron nitride (h-BN) is a stable crystalline form consisting of equal numbers of boron and nitrogen atoms in a honeycomb arrangement [4] 6 . As it has considerably high thermal and chemical stabilities 7], it can be used for high-temperature applications. The BN nanotube (BNNT) [8, 9] is a nano-sized seamless cylinder that can be considered a rolled-up h-BN sheet. It also has a wide band gap that is almost independent of the tube diameter, and chirality [10]. For $s p^{2}$-bonded carbon nanostructures such as fullerenes, carbon nanotubes and graphene, the Stone-Wales (SW) transformation [11] is believed to introduce topological defects or isomerization, which results in a $90^{\circ}$ rotation of two carbon atoms with respect to the midpoint of the $\mathrm{C}-\mathrm{C}$ bond.

When an SW defect is present in h-BN or BNNT, B$\mathrm{B}$ and $\mathrm{N}-\mathrm{N}$ bonds should be created, and the formation of these bonds increases the total energy of the system. Thus, the topological defect created by the SW rearrangement is lacking. However, the formation of B-B and N-N bond pairs can be avoided if there is a C-C pair defect in h-BN (or BNNT). Using post-synthesis doping, carbon impurities can be substituted in the h-BN layers 12 14]. During the formation of BNNTs and h$\mathrm{BN}$ sheets using the chemical vapor deposition (CVD), carbon atoms may act as substitutional defects, because residual hydrocarbon may remain in the chamber, or carbon impurities are solubilized in the metal catalyst. If carbon substitutional atoms congregate together in a local area in h-BN (or a bundle of BNNTs) by segregation, they may form the interlayer (or intertube) conduction channels. As mentioned above, the C-C pair defect in the h-BN network prohibits the formation of B-B and $\mathrm{N}-\mathrm{N}$ bonds during the SW transformation. As the SW-transformed defects may affect the mechanical and electrical properties of the BN nanostrucutres, we should thus study these defects in the C-doped BN sheets or BNNTs. This work may explain the formation of topological defects in the carbon-doped BNNTs or h-BN layers that can be produced in the CVD processes. In this paper, we present our first-principles study of the structural and electronic properties of the BNNTs containing two substitutional carbon defects. The effect of carbon doping on the SW transformation is also examined.

We carried out total energy calculations for our model systems based on the density functional theory [15]. The local density approximation (LDA) 16] with spin polarization was used for the exchange-correlation functional. Some calculations were repeated using the generalized gradient approximation. We found that the exchangecorrelation functional did not change our main conclusion that the SW defect in the C-doped BNNT may be ob-

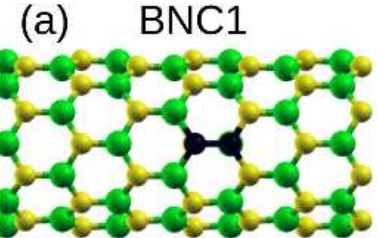

(c) BNCR1

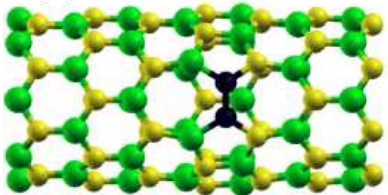

(e) BNR1

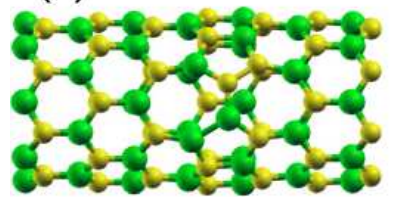

(b) $\mathrm{BNC2}$

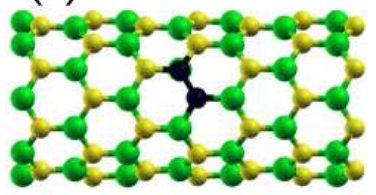

(d) BNCR2
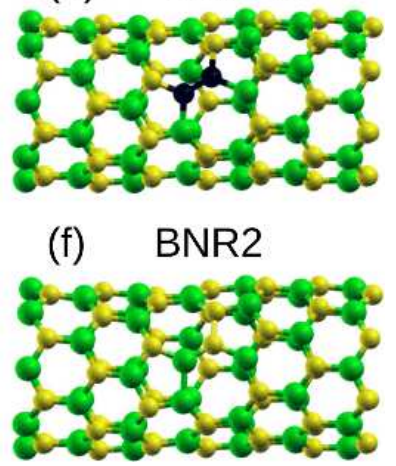

(f) BNR2

FIG. 1: Optimized structures of $(8,0)$ BNNTs with a C-C pair substitutional defect or the SW defects. (a) The BNNT with a C-C pair substitutional defect parallel to the tube axis, (b) the BNNT with a C-C pair substitutional defect with an oblique angle with respect to the tube axis. (c) and (d) show the SW-transformed C-C pair defect from (a) and (b), respectively. (e) and (f) are the BNNTs with SW defects which are parallel and tilted to the tube axis, respectively. Yellow, green and black balls represent nitrogen, boron and carbon atoms, respectively. 
served with a higher probability than in the bare BNNT. To study the energetics and the activation energy barrier in the SW transformation of the systems, we used a plane wave basis set with an energy cutoff of $400 \mathrm{eV}$ and ultrasoft pseudopotentials 17 implemented in the Vienna ab initio simulation package (VASP) 18, 19]. The atomistic models were relaxed until the residual forces on the atoms became smaller than $0.03 \mathrm{eV} / \AA$. For the activation energy barrier, we employed the nudged elastic band method[20]. Eight replicas were selected, including the initial and final configurations to construct an elastic band. To plot the density of states (DOS) and the wavefunctions with phase information, we used the OpenMX code 21]. In the calculations using the OpenMX code, the ionic potentials were described with norm-conserving Troullier-Martins pseudopotentials. Wavefunctions were expanded in a pseudoatomic orbital basis set 22. with an energy cutoff of $120 \mathrm{Ry}$. As a model system, we chose the $(8,0)$ zigzag BNNT containing $48 \mathrm{~B}$ atoms and $48 \mathrm{~N}$ atoms. The supercell size in the lateral direction was 25 $\AA$ to avoid the interaction between the defects in neighboring BNNTs, and the size in the axial direction was $12.96 \AA$.

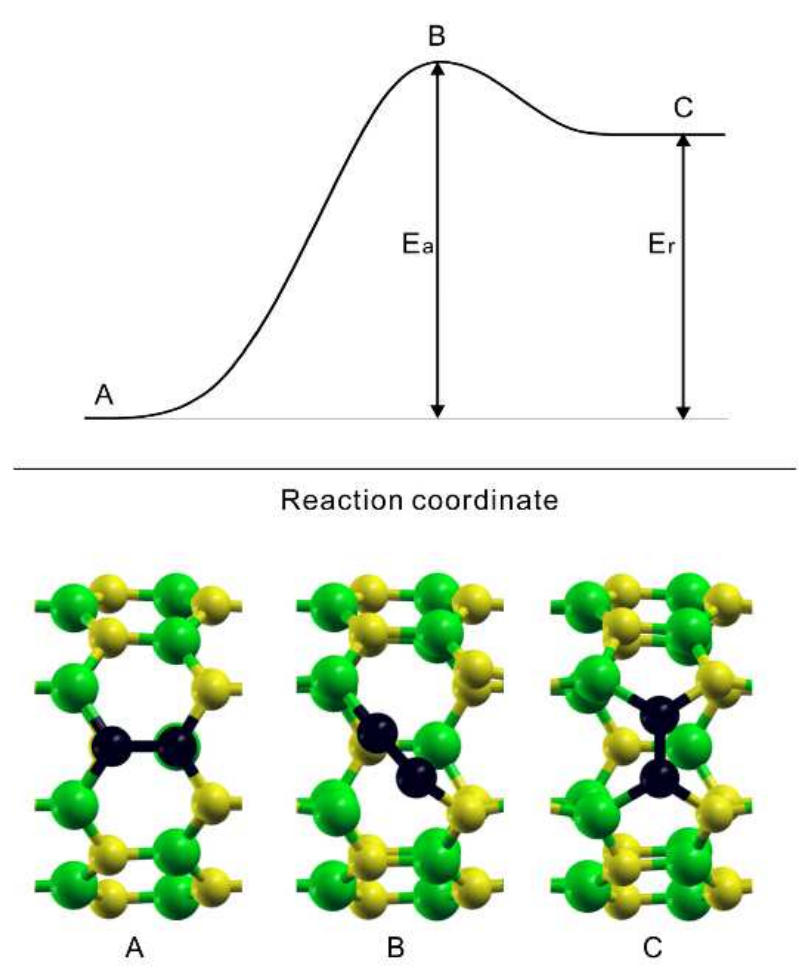

FIG. 2: (Top panel) Energy profile along the reaction coordinate in the SW transformation of BNNTs with a C-C pair substitutional defect or the SW defects. The states "A", "B", and "C" represent the initial, transition, and final states in the SW transformations, respectively. The activation and formation energies are denoted as $E_{a}$ and $E_{r}$, respectively. (Bottom panel) The corresponding three states are shown for the SW transformation from BNC1 to BNCR1.
First, we optimized the C-doped and undoped BNNTs. The perfect BNNT has a B-N bond length of $\sim$ $1.44 \AA$. Our total energy calculations show that the two substitutional carbon atoms tend to bind to each other. This finding agrees with K. Yuge's report that the lowestformation-energy structures for monolayer boron-carbonnitride favor $\mathrm{B}-\mathrm{N}$ and $\mathrm{C}-\mathrm{C}$ bonds whereas they disfavor B-C, C-N, B-B, and N-N bonds 23]. For C-doping, two types of $\mathrm{C}-\mathrm{C}$ pairs can be considered. As shown in Figure 1, one pair (BNC1) was parallel to the tube axis and the other (BNC2) had an oblique angle with the tube axis. The bond length of the C-C pair was $1.37 \AA(1.39 \AA)$ for BNC1 (BNC2). Note that the bond length of benzene $\left(\mathrm{C}_{6} \mathrm{H}_{6}\right)$ has a very similar C-C bond length of $1.40 \AA$. Although the model of $\mathrm{BNC} 1$ had the lowest energy among our model systems for the C-C pair, the energy difference between $\mathrm{BNC} 1$ and $\mathrm{BNC} 2$ was only $\sim 1 \mathrm{meV} /$ atom. Thus, two structures may coexist. In fact, by the chemical potential analysis, we found that the formation of carbon substitutional defects in the BNNTs, BNC1 or $\mathrm{BNC} 2$, is possible when the chemical potential of an isolated $\mathrm{C}-\mathrm{C}$ pair is larger than by $-18.02 \mathrm{eV}$ or $-17.90 \mathrm{eV}$. Note that the energy of an C-C pair is $-10.09 \mathrm{eV}$.

In the $\mathrm{C}-\mathrm{C}$ pair, the $\mathrm{C}$ atom bonded to two $\mathrm{B}$ atoms protruded out by $\sim 0.1 \AA$. The B-C bond length was 1.52 $\AA$ and the $\mathrm{N}-\mathrm{C}$ bond length was $1.46 \AA$, which was due to the longer covalent radius of $\mathrm{B}\left(\mathrm{r}_{\text {covalent }, B}=0.84 \AA\right)$ than that of $\mathrm{N}\left(\mathrm{r}_{\text {covalent }, N}=0.71 \AA\right)$. When the $\mathrm{C}-\mathrm{C}$ bond was rotated by $90^{\circ}$, the bond length slightly decreased to 1.35 $\AA$. Here, the B-C bond length was increased to $1.61 \AA$, and the $\mathrm{N}-\mathrm{C}$ bond length was slightly decreased to 1.44 $\AA$. After the SW transformation from the BNC1 to the BNCR1 structure, the total energy of BNCR1 increased by $2.5 \mathrm{eV}$, compared to that of BNC1. For this structure, the activation energy from BNC1 to BNCR1 was $5.5 \mathrm{eV}$. Similarly, the formation and activation energies were 2.2 $\mathrm{eV}$ and $5.6 \mathrm{eV}$, respectively, for the $\mathrm{SW}$ transformation from BNC2 to BNCR2 (see Table I).

For comparison, we also calculated the formation and activation energies for the SW transformation in the undoped $(8,0)$ BNNT as shown in Figure 2. For the SW transformation from $\mathrm{BN} 1$ to $\mathrm{BNR} 1$ (or from $\mathrm{BN} 2$ to BNR2), the formation energy was $5.6 \mathrm{eV}(5.7 \mathrm{eV})$, and

TABLE I: Calculated activation $\left(E_{a}\right)$ and formation $\left(E_{r}\right)$ energies for the SW transformation from the C-doped or the BNNTs to the BNNTs with SW defects.

\begin{tabular}{ccc}
\hline & $E_{a}(\mathrm{eV})$ & $E_{r}(\mathrm{eV})$ \\
\hline $\mathrm{BNC} 1 \rightarrow$ BNCR1 & 5.50 & 2.51 \\
$\mathrm{BNC} 2 \rightarrow$ BNCR2 & 5.64 & 2.17 \\
$\mathrm{BN} 1 \rightarrow$ BNR1 & 7.04 & 5.61 \\
$\mathrm{BN} 2 \rightarrow$ BNR2 & 7.63 & 5.72 \\
\hline
\end{tabular}


the activation energy was $7.0 \mathrm{eV}(7.6 \mathrm{eV})$. Here, a B-B bond and a N-N bond are produced, and their lengths are $1.73 \AA$ (B-B) and $1.47 \AA(\mathrm{N}-\mathrm{N})$, respectively. We found that the rotating $\mathrm{C}-\mathrm{C}$ bond $(1.24 \AA)$ was shorter than the rotating B-N bond (1.29 $\AA$ ) in the transition states. This implies that the shorter bond results in a lower activation energy barrier during the SW transformation in a small area. The high formation energy is due to the unstable $\mathrm{B}-\mathrm{B}$ and $\mathrm{N}-\mathrm{N}$ bonds as mentioned earlier. Interestingly, electrons were accumulated in the $\mathrm{N}$ atom of the rotating B-N pair in the transition state for the undoped BNNT. In addition, our charge analysis revealed that electron donation from the $\mathrm{C}$-C pair defect in the C-doped BNNT slightly weakens the dangling-bond-like character of the $\mathrm{B}$ and $\mathrm{N}$ atoms in the transition configuration. In our previous study of the SW transformation in $\mathrm{M}_{0} \mathrm{C}_{60}(\mathrm{M}$ $=\mathrm{K}, \mathrm{Ca}$, and $\mathrm{La}$ ), we similarly observed that the electron donation by the incorporated metal atom lowers the activation energy barrier 24. These findings can explain the lower activation energy barrier of the $\mathrm{C}-\mathrm{C}$ pair defect compared to that of the B-N pair. Therefore, when the C-doped BNNT does exist, e.g., from the sample preparation, we can conclude that the probability that the SW defect is observed is higher in the C-doped BNNT than in the undoped BNNT.

To check a charge doping effect, we calculated doping of a hole (charge $+e$ state) for all the model structures (BNR1, BNR2, BNCR1, and BNCR2), and an electron doping case (charge - $e$ state) for BNR2 structure. When a hole was doped, the activation energy barriers were reduced from $7.04 \mathrm{eV}$ to $6.91 \mathrm{eV}$ for BNR1, $7.63 \mathrm{eV}$ to 7.18 $\mathrm{eV}$ for BNR2, $5.50 \mathrm{eV}$ to $5.23 \mathrm{eV}$ for BNCR1, and 5.64 $\mathrm{eV}$ to $5.52 \mathrm{eV}$ for BNCR2, respectively. For an electron doping in BNR2, and the activation energy was decreased from $7.63 \mathrm{eV}$ to $6.69 \mathrm{eV}$. Therefore, we can conclude that charge doping affects the activation energy barriers of the SW transformation.

In a growth process of the BNNTs, carbon atoms may be incorporated as residual impurities. Or one can produce carbon substitutional defects in the BNNTs on purpose for doping. Our results demonstrate that two carbon atoms far from each other tend to become closer and form a C-C pair. The energy difference between the C-C pair and two separate carbon atoms was larger than $5 \mathrm{eV}$ in our LDA calculation. On the other hand, some metal atoms or molecules such as $\mathrm{O}_{2}$ and $\mathrm{Br}_{2}$ may be adsorbed in the vicinity of the $\mathrm{C}-\mathrm{C}$ pair. Then, electron transfer takes place between the adsorbate and the nanotube. As mentioned above, charge doping enables the activation energy barrier to become lowered and the probability of the SW transformation to become higher.

According to our LDA calculation, the energy band gap of the perfect $(8,0) \mathrm{BNNT}$ is $3.6 \mathrm{eV}$ as shown in Figure 3a. R. Wu et al. reported that the BNNT could have a magnetic moment due to the carbon doping [25]. They considered only one carbon atom in the supercell.
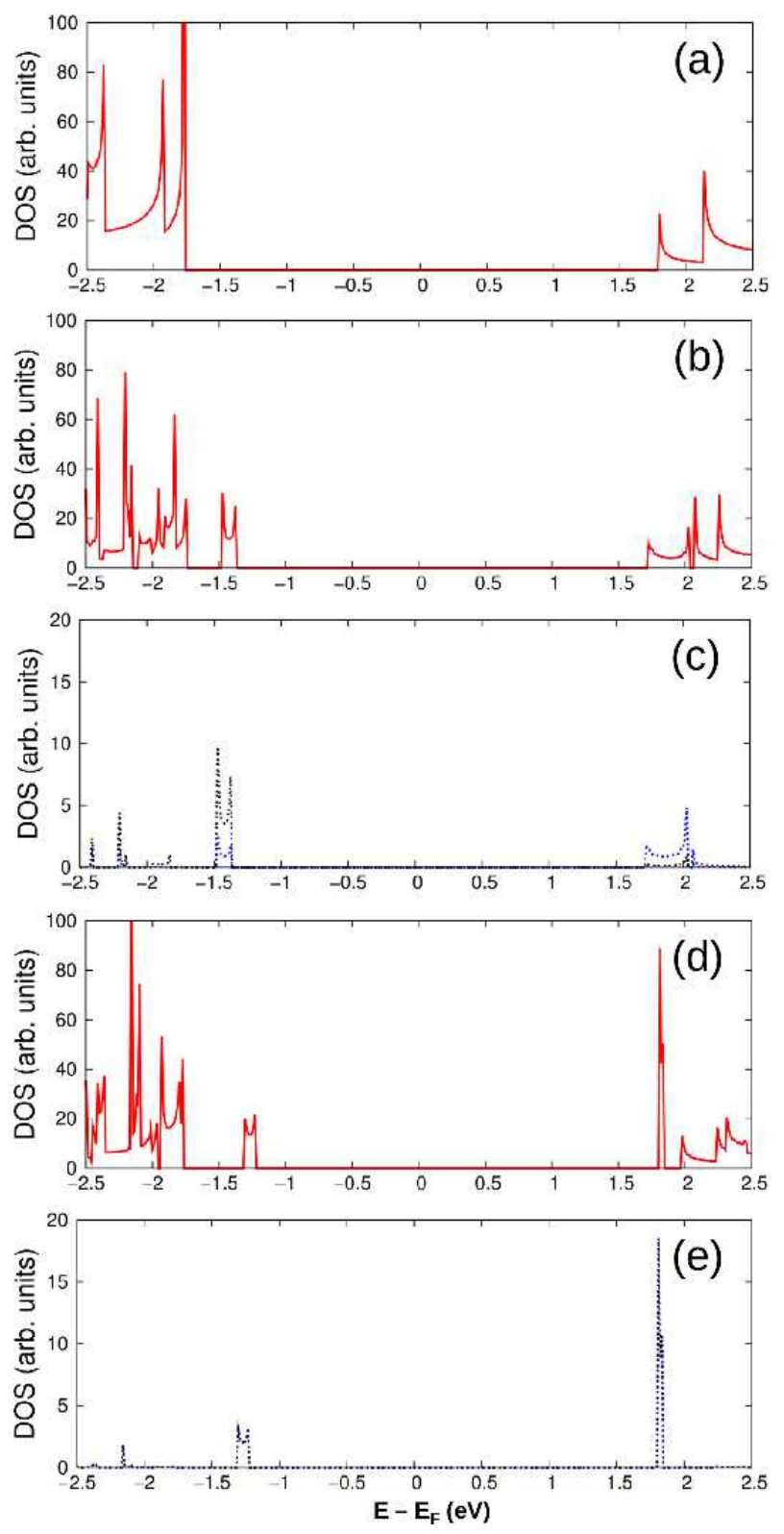

FIG. 3: (a) Total DOS of the perfect $(8,0)$ BNNT. Total DOS of BNNTs with (b) the C-C pair substitutional defect or (d) the SW defects. Dotted lines in (c) and (e) indicate the projected DOS of two $\mathrm{C}$ atoms in the $\mathrm{C}-\mathrm{C}$ pair defect in each case.

However, our calculational results demonstrated that the magnetic moment does not occur in the BNNT with the C-C pair defect. According to a recent report by N. Berseneva et al., the magnetic moment is zero when the numbers of carbon atoms replaced by nitrogen and boron atoms are identical[26]. Figure 30 shows the total DOS of BNNTs with the C-C pair substitutional defect (e.g., BNC1), which clearly demonstrates that a few localized states occur in the band gap in the presence of the C-C pair defect. As shown in Figure 35 , the projected DOS of the two $\mathrm{C}$ atoms in the $\mathrm{C}-\mathrm{C}$ pair defect indicates that 
the gap states originate from the substitutional carbon pair defects. When a $\mathrm{C}-\mathrm{C}$ pair forms a $\mathrm{SW}$ defect by a $90^{\circ}$ rotation as seen in BNCR1, the electronic states originating from the SW defect have flatter bands with more localized characters. These characteristics are reflected in the sharp and narrow peaks in the DOS in the gap (Figure 3d and 3e).

Figure 4 shows wavefunction characters resulting from the defect states in the BNNTs with the substitutional carbon pair defect and its SW-transformed defect. The occupied state at $-1.4 \mathrm{eV}$ and the unoccupied state at $+1.7 \mathrm{eV}$ shown in Figure 4 a are the localized defect states originating primarily from the $\mathrm{C}-\mathrm{C}$ pair of the $\mathrm{BNC} 1$ structure. The filled state has a bonding character, whereas the unfilled state has a antibonding character at the $\mathrm{C}-\mathrm{C}$ pair. As displayed in Figure 4 a, the electron density at the $\mathrm{C}$ atom bonded to $\mathrm{B}$ atoms is somewhat higher than that at the $\mathrm{C}$ atom bonded to $\mathrm{N}$ atoms at $-1.4 \mathrm{eV}$. Additionally, the B-C bonds are in the bonding states but the $\mathrm{C}-\mathrm{N}$ bonds are in the antibonding states. On the other hand, Figure $4 \mathrm{~b}$ shows the localized defect states at $-1.3 \mathrm{eV}$ and $+1.8 \mathrm{eV}$ from the $\mathrm{SW}$-transformed $\mathrm{C}-\mathrm{C}$ pair defect of the BNCR1 structure. Similar to the BNC1 case, bonding and antibonding characters of the carbon pair appear at $-1.3 \mathrm{eV}$ and $+1.8 \mathrm{eV}$, respectively. Note that there is no mirror (reflection) symmentry for the BNNT with respect to the plane containing the midpoint of the $\mathrm{C}-\mathrm{C}$ bond. However, the mirror symmetry is manifest with respect to the planes containing the $\mathrm{C}$ $\mathrm{C}$ bond itself (as in BNC1) as well as the midpoint of the SW-transformed C-C bond (as in BNCR1). These features were retained for two localized states as shown in Figure $4 \mathrm{a}$ and also for a localized state at $-1.3 \mathrm{eV}$ as
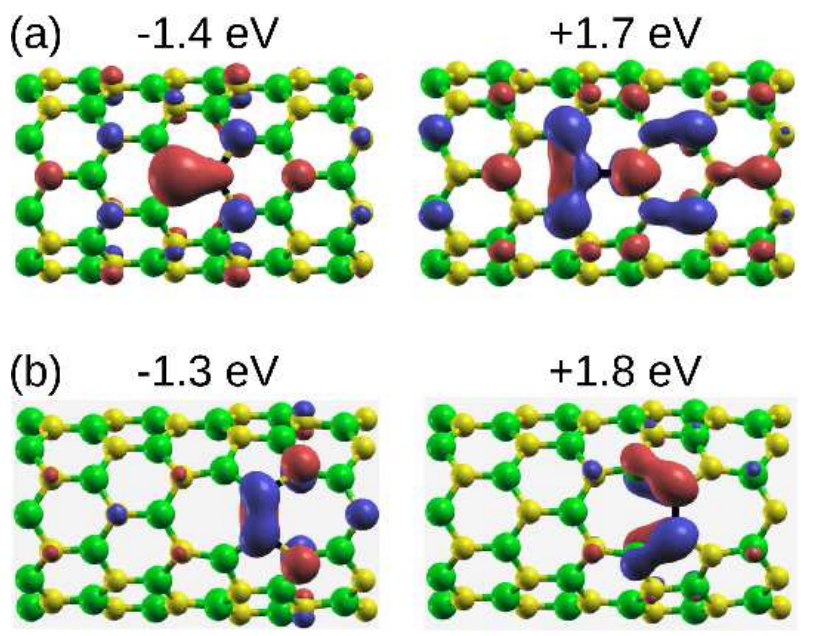

FIG. 4: Wavefunctions originating from the defect states in the BNNTs with (a) the substitutional C-C pair defect (BNC1) and (b) the SW-transformed C-C pair defect (BNCR1). Red and blue colors represent the opposite signs in the wavefunctions at the $\Gamma$-point. shown in Figure 40 .

In summary, we have introduced substitutional carbon pair defects in BNNTs to examine the modifications in the structural and electronic properties of the nanotube. Our results demonstrate that the two carbon atoms prefer to bind to each other with a bond length of $\sim 1.37$ $\AA$. For the SW transformation of the C-C pair defect, the formation energy of the SW defect is approximately $2.5 \mathrm{eV}$ and the activation energy is approximately 5.5 $\mathrm{eV}$. Both the formation and activation energies of the $\mathrm{C}-\mathrm{C}$ bond are much lower than those of the B-N bond in the undoped BNNT in the SW transformation, which are $\sim 5.5 \mathrm{eV}$ and $\sim 7.0 \mathrm{eV}$, respectively. Consequently, it would be very difficult to find a topological defect such as the SW defect in the perfect BNNT. Conversely, such a topological defect could be observed with a higher probability in the C-rich defective BNNTs.

\section{Acknowledgments}

This research was supported by the Priority Research Center Program (2011-0018395), the Converging Research Center Program (2011K000620), and the Basic Science Research Program (KRF-2008-313-C00217) through the National Research Foundation of Korea (NRF) funded by the Ministry of Education, Science and Technology(MEST). G. K. acknowledges support by the Basic Science Research Program through MEST/NRF (2010-0007805).

[1] Y.N. Xu, W.Y. Ching, Phys. Rev. B 44 (1991) 7787.

[2] M.S. Si, D.S. Xue, Phys. Rev. B 75 (2007) 193409.

[3] X. Blase, A. Rubio, S.G. Louie, M.L. Cohen, Europhys. Lett. 28 (1994) 335.

[4] K. Watanabe, T. Taniguchi, H. Kanda, Nature Mater. 3 (2004) 404.

[5] A. Rubio, J.L. Corkill, M. L. Cohen, Phys. Rev. B 49 (1994) 5081.

[6] N.G. Chopra, R.J. Luyken, K. Cherrey, V.H. Crespi, M.L. Cohen, S. G. Louiea, A. Zettl, Science 269 (1995) 966.

[7] D. Golberg, Y. Bando, C.C. Tang, C.Y. Zhi, Adv. Mater. 19 (2007) 2413.

[8] A. Loiseau, F. Willaime, N. Demoncy, G. Hug, H. Pascard, Phys. Rev. Lett. 76 (1996) 4737.

[9] D. Golberg, Y. Bando, W. Han, K. Kurashima, T. Sato, Chem. Phys. Lett. 308 (1999) 337.

[10] G.G. Fuentes, E. Borowiak-Palen, T. Pichler, X. Liu, A. Graff, G. Behr, R.J. Kalenczuk, M. Knupfer. J. Fink, Phys. Rev. B 67 (2003) 035429.

[11] A.J. Stone, D.J. Wales, Chem. Phys. Lett. 128 (1986) 501.

[12] O.L. Krivanek et al., Nature (London) 464 (2010) 571.

[13] X. Wei, M. Wang, Y. Bando, D. Golberg, J. Am. Chem. Soc. 132 (2010) 13592. 
[14] X. Wei, M. Wang, Y. Bando, D. Golberg, ACS Nano 5 (2011) 2916.

[15] P. Hohenberg, W. Kohn, Phys. Rev. 136 (1964) B864.

[16] W. Kohn, L.J. Sham, Phys. Rev. 140 (1965) A1133.

[17] D. Vanderbilt, Phys. Rev. B 41 (1990) 7892.

[18] G. Kresse, J. Hafner, Phys. Rev. B 47 (1993) R558.

[19] G. Kresse, J. Furthmüller, Phys. Rev. B 54 (1996) 11169.

[20] G. Mills, H. Jónsson, Phys. Rev. Lett. 72 (1994) 1124.

[21] T. Ozaki, Phys. Rev. B. 64 (2001) 195110.
[22] T. Ozaki, Phys. Rev. B. 67 (2003) 155108.

[23] K. Yuge, Phys. Rev. B 79 (2009) 144109.

[24] W.I. Choi, G. Kim, S. Han, J. Ihm, Phys. Rev. B 73 (2006) 113406.

[25] R.Q. Wu, L. Liu, G.W. Peng, Y.P. Feng, Appl. Phys. Lett. 86 (2005) 122510.

[26] N. Berseneva, A.V. Krasheninnikov, R.M. Nieminen, Phys. Rev. Lett. 107 (2011) 035501. 